\title{
Nitrogen and Phosphorus Self-Doped Carbon Supported Palladium as Highly Efficient Electrocatalysts for Formic Acid Oxidation
}

Chenxi Lu ${ }^{1,2}$, Duo $\mathrm{Yu}^{1,2}$, Zhiguo Yan ${ }^{1,2}$, Xiaojun Yang ${ }^{1,2}$, Wei Yang ${ }^{1,4, *}$, Zaosheng Lv $^{3}$, Zhengfang Tian ${ }^{5, *}$ and Qifeng Tian ${ }^{1,2, *}$

${ }^{1}$ Key Laboratory of Green Chemical Process of Ministry of Education \& Hubei Key Laboratory of Novel Reactor and Green Chemical Technology (Wuhan Institute of Technology), Wuhan 430205, People's Republic of China

${ }^{2}$ School of Chemical Engineering and Pharmacy, Wuhan Institute of Technology, Wuhan 430205, People's Republic of China

${ }^{3}$ Key Laboratory of Hubei Province for Coal Conversion and New Carbon Materials, Wuhan University of Science and Technology, Wuhan 430081, People's Republic of China

${ }^{4}$ School of Chemistry and Environmental Engineering, Wuhan Institute of Technology, Wuhan 430205, People's Republic of China

${ }^{5}$ College of Chemistry and Chemical Engineering, Hubei Key Laboratory for Processing and Application of Catalytic Materials, Huanggang Normal University, Huanggang 438000, People's Republic of China

*E-mail: qftian@wit.edu.cn (Q. T. ), yangweight@126.com (W. Y. ), tzf7801@163.com (Z. T.)

doi: $10.20964 / 2021.05 .37$

Received: 16 November 2020 / Accepted: 23 January 2021 / Published: 31 March 2021

Exploring new Pd-based catalysts with both high activity and $\mathrm{CO}$ resistance for formic acid oxidation remains of great importance of direct formic acid fuel cell (DFAFC) technology. Here, a nitrogen and phosphorus self-doped carbon have been facilely synthesized by a direct pyrolysis of aminophosphonic acid resin (APAR) which was employed as the N, P and C sources. The synthesized N, P self-doped carbon at different temperatures (NPC-T, $\mathrm{T}=850,900,950,1000{ }^{\circ} \mathrm{C}$ ) were used as supports for the preparation of Pd/NPC-T electrocatalysts by microwave-assisted glycol reduction method. All prepared catalysts show better catalytic performance than commercial $\mathrm{Pd} / \mathrm{C}$ counterparts towards formic acid electro-oxidation (FAEO). Among them, Pd/NPC-900 demonstrates optimal electrocatalytic activity with an enhancing factor of 1.98 than commercial $\mathrm{Pd} / \mathrm{C}$ catalyst. Moreover, the Pd/NPC-T catalysts present excellent stability, highly tolerant to $\mathrm{CO}$ poisoning and faster charge-transfer kinetics of FAEO. The superior catalytic performance of the Pd/NPC-T catalysts are attributed to the doping of nitrogen 
and phosphorus, which modified the electronic structure of Pd particles and accelerated the removal of CO intermediates.

Keywords: N, P self-doped carbon; Pd electrocatalyst; Formic acid oxidation; Microwave-assisted reduction

\section{$\underline{\text { FULL TEXT }}$}

(C) 2021 The Authors. Published by ESG (www.electrochemsci.org). This article is an open access article distributed under the terms and conditions of the Creative Commons Attribution license (http://creativecommons.org/licenses/by/4.0/). 